\title{
Naimark-Sacker Bifurcations in Linearly Coupled Quadratic Maps
}

\author{
Paulo C. Rech ${ }^{\mathrm{a}, \mathrm{b}, *}$, Marcus W. Beims ${ }^{\mathrm{b}}$, Jason A. C. Gallas $\mathrm{b}, \mathrm{c,d}$ \\ ${ }^{a}$ Departamento de Física, Universidade do Estado de Santa Catarina, \\ 89223-100 Joinville, Brazil. \\ b Departamento de Física, Universidade Federal do Paraná, \\ 81531-990 Curitiba, Brazil. \\ ${ }^{\mathrm{c}}$ Instituto de Física, Universidade Federal do Rio Grande do Sul, \\ 91501-970 Porto Alegre, Brazil. \\ ${ }^{\mathrm{d}}$ Institut für Computer Anwendungen, Universität Stuttgart, \\ Pfaffenwaldring 27, D-70569 Stuttgart, Germany.
}

\begin{abstract}
We report exact analytical expressions locating the $0 \rightarrow 1,1 \rightarrow 2$ and $2 \rightarrow 4$ bifurcation curves for a prototypical system of two linearly coupled quadratic maps. Of interest is the precise location of the parameter sets where Naimark-Sacker bifurcations occur, starting from a non-diagonal period- 2 orbit. This result is the key to understand the onset of synchronization in networks of quadratic maps.
\end{abstract}

Key words: Synchronization, Quasiperiodicity, Naimark-Sacker Bifurcation PACS: 05.45.Pq, 05.45.Xt, 02.30.Oz

An essential clue to understand how dynamical systems synchronize and the origin of their riddled basins of attraction is provided by the mechanisms underlying the transverse desestabilization of low periodic orbits embedded in chaotic attractors $[1,2,3,4]$. So far, these mechanisms have been investigated only via direct numerical simulation of the dynamics. The purpose of this paper is to report exact analytical results for a prototypic model, two linearly coupled quadratic maps, defined by the following pair of equations

$$
x_{t+1}=a-x_{t}^{2}+b\left(x_{t}-y_{t}\right), \quad y_{t+1}=a-y_{t}^{2}+b\left(y_{t}-x_{t}\right) .
$$

Here $x_{t}, y_{t}$ represent dynamical variables, $a$ is the local nonlinearity, $b$ the coupling, and $t=0,1,2, \ldots$ is the discrete time. Systems of coupled nonlinear

\footnotetext{
* Corresponding author

Email address: rech@fisica.ufpr.br (Paulo C. Rech).
} 
oscillators are nowadays used in practical applications, e.g., in the investigation of coupled p-n junctions [5] and of Josephson-junction arrays [6]. They also serve as useful approximations of nonlinear partial differential equations describing, for example, the onset of fluid turbulence [7] and reaction diffusion systems [8]. Previous analytical results were obtained using linear stability analysis $[9,10,11]$, and renormalization theory[12]. Here we go well beyond this presenting (i) the algebraic varieties delimiting stability domains for motions of periods 1 and 2, and, (ii) the Naimark-Sacker line (also called Hopf bifurcation line) showing where quasiperiodicity stabilizes following a period-2 oscillation. The motivation for considering this problem anew is our interest in using more realistic and flexible maps as local oscillators in applications dealing with diffusive-advective effects like, e.g. in ocean convection $[13,14,15]$. For additional discussion of problems involving coupled systems we refer the reader to the excellent tutorials in Refs. $[1,2,3,4]$.

As is well-known[16,17], there are three ways in which a fixed point $p$ of a discrete map $f$ may fail to be hyperbolic: $D f(p)$ may have an eigenvalue +1 , an eigenvalue -1 , or a pair of complex eigenvalues, say $\lambda$ and $\bar{\lambda}$, with $|\lambda|=1$. The first two situations are familiar from period-doubling cascades and appear much more frequently than the last one. But it is precisely this last situation, characteristic of a Naimark-Sacker bifurcation, that is of great interest for coupled systems and that we consider here.

Equation (1) has four fixed points, two diagonal and two non-diagonal:

$$
D_{1} \equiv\left(x_{1}, x_{1}\right), \quad D_{2} \equiv\left(x_{2}, x_{2}\right), \quad N_{1} \equiv\left(x_{3}, x_{4}\right), \quad N_{2} \equiv\left(x_{4}, x_{3}\right),
$$

where $x_{1}=(-1+\xi) / 2, x_{2}=(-1-\xi) / 2, x_{3}=(2 b-1+\eta) / 2, x_{4}=(2 b-1-\eta) / 2$, and $\xi=\sqrt{1+4 a}$ and $\eta=\sqrt{1+4 a-4 b^{2}}$. The stability of these points is ruled by the equation $|J-\lambda I|=0$, where $\lambda$ is the eigenvalue, $I$ is the identity matrix and $J$ is the Jacobian matrix of the mapping. The eigenvalues of these four fixed points are always real, with $D_{2}, N_{1}$ and $N_{2}$ being always unstable since at least one of their eigenvalues is larger than 1 . The remaining fixed point, $D_{1}$, is stable in the region delimited by $-1 / 4<a<3 / 4$ and $\sqrt{1+4 a} / 2-1<$ $b<\sqrt{1+4 a} / 2$, bounded by the curves

$$
a=-\frac{1}{4}, \quad a=\frac{3}{4}, \quad c_{1} \equiv 4 a-4 b^{2}+1=0, \quad c_{2} \equiv 4 a-4 b^{2}-3-8 b=0,
$$

shown in Fig. 1.

The stable fixed point bifurcates into two quite different period-2 orbits when one increases $a$, depending on the value of $b$. When crossing the line $a=3 / 4$ one finds diagonal period-2 orbits with signature $(u, u) \rightarrow(v, v) \rightarrow(u, u) \rightarrow$ $\ldots$, where $u=(1+\sqrt{-3+4 a}) / 2$ and $v=(1-\sqrt{-3+4 a}) / 2$. We denote such orbits by $P 2 D$. Beyond the curve $c_{2}=0$ one finds non diagonal orbits with a signature $(s, t) \rightarrow(t, s) \rightarrow(s, t) \rightarrow \ldots$ where $s=(2 b+1+$ 


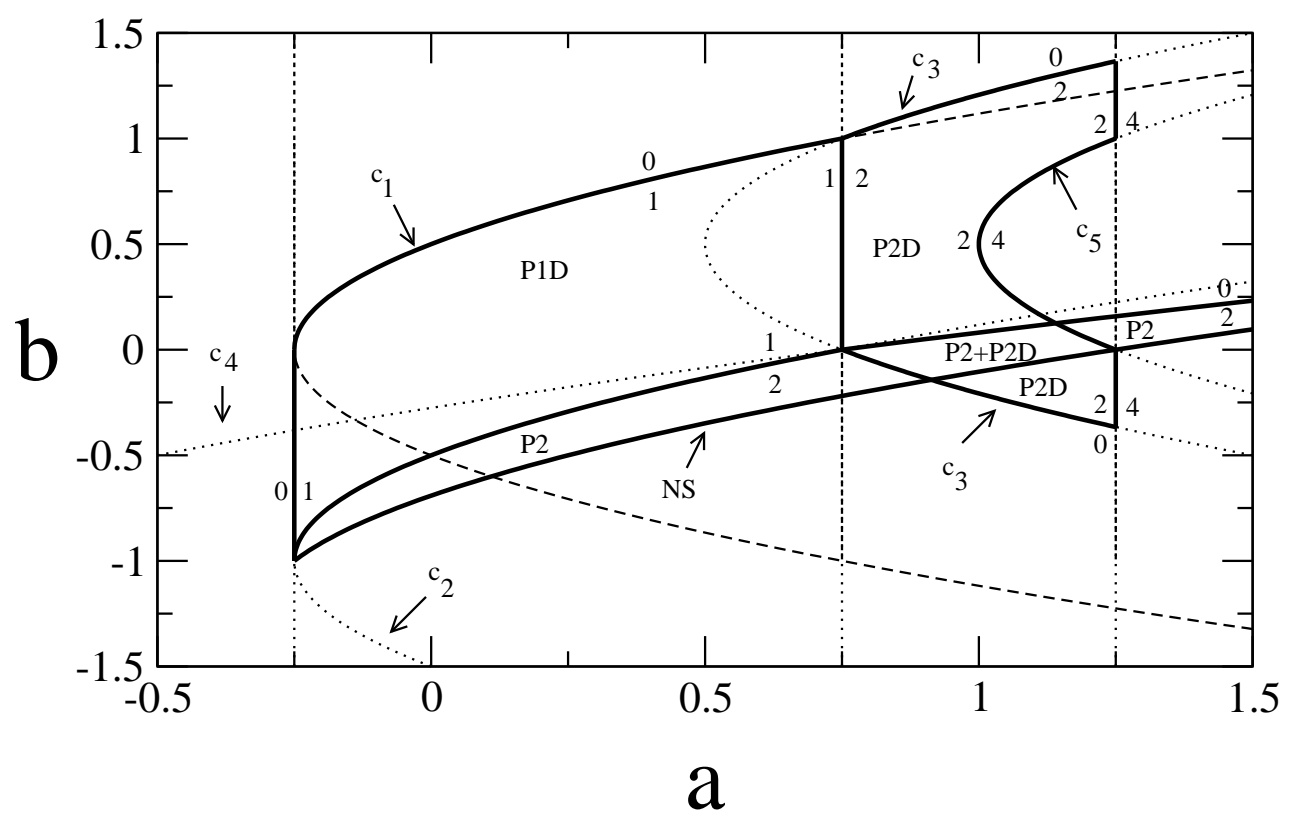

Fig. 1. Stability domains for period-1 and period-2 orbits. $P 2 D$ and $P 2$ refer to domains of diagonal and non-diagonal orbits, respectively. Numbers indicate periods. $\left.\sqrt{4 a-4 b^{2}-8 b-3}\right) / 2$ and $t=\left(2 b+1-\sqrt{4 a-4 b^{2}-8 b-3}\right) / 2$. These we represent by $P 2$.

The $P 2 D$ orbit has real eigenvalues and is stable in the domain delimited by the curves

$$
a=\frac{3}{4}, \quad a=\frac{5}{4}, \quad c_{3} \equiv 4 a-4 b^{2}-3+4 b=0, \quad c_{5} \equiv 4 a-4 b^{2}-5+4 b=0 .
$$

It loses stability along $c_{5}=0$ or $a=5 / 4$, after a $2 \rightarrow 4$ bifurcation. There is a $P 4 D$ diagonal orbit when crossing the line $a=5 / 4$, and a $P 4$ non-diagonal orbit when crossing $c_{5}=0$.

The eigenvalues of the $P 2$ orbit are

$$
\lambda_{ \pm}=6 b^{2}+10 b-4 a+4 \pm 2 b \sqrt{5 b^{2}+10 b-4 a+4}
$$

and can be complex. From these expressions we obtain[16] the following formula for the Naimark-Sacker bifurcation line:

$$
a_{N S}=b^{2}+\frac{5}{2} b+\frac{5}{4} .
$$

It is important to observe that the linear coupling of the oscillators was fundamental in allowing the derivation of the above analytical result. Already a 


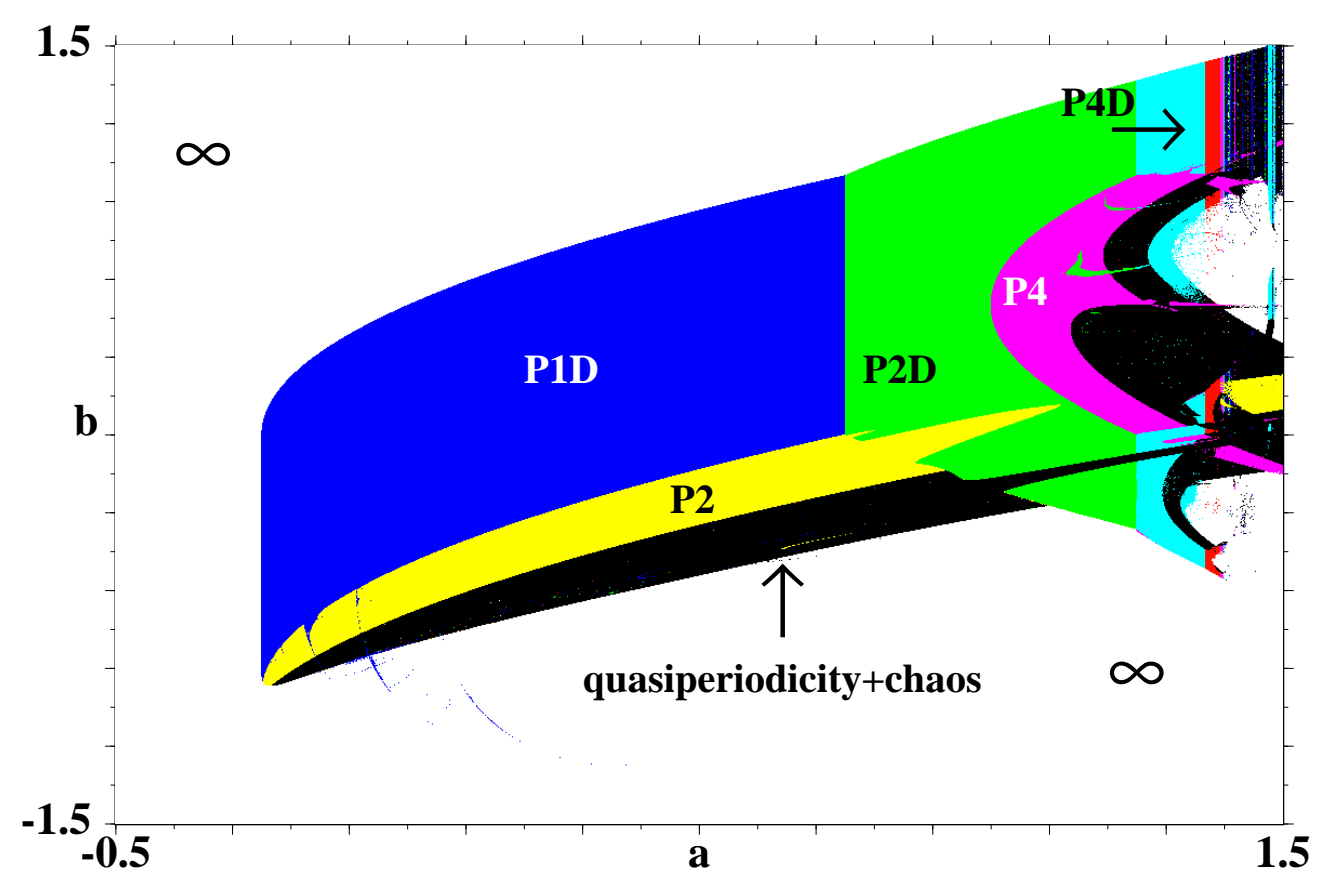

Fig. 2. Stability domains obtained by discretizing the parameter interval in a mesh of $1200 \times 600$ points. Different gray shadings represent stability domains of attractors with different periods.

quadratic coupling generates formulas which are intractable with present day hardware and commercially available software.

From the eigenvalues along $c_{2}=0$ as well as the condition $\lambda_{1}=\lambda_{2}=1$ we find that: (i) for $b>0$, an unstable P2 orbit is born along $c_{2}=0$ stabilizing however along $c_{4}=4 a-4 b^{2}-3-12 b=0$, and (ii) for $-1<b<0$, a stable P2 orbit is born, also along $c_{2}=0$. Figure 1 summarizes all period-2 bifurcation loci. In the small diamond-shaped region in Fig. 1, delimited by $c_{3}=0, c_{4}=0$, $c_{5}=0$ and the Naimark-Sacker line (indicated by NS), there is coexistence of $P 2$ and $P 2 D$ motions. On the NS line the $P 2$ orbit disappears, to give rise to two closed curves (limit cycles).

The stability domains for the coupled quadratic maps can be generated numerically, using the method described in Ref. [18], and they are shown in Fig. 2, displaying the richness of the several coexisting motions in parameter space. In this figure the basin of unbounded (diverging) attractors is indicated by $\infty$. The lower border line seen between the P2 domain and black domain is defined by the Naimark-Sacker line. This means that inside the black domain, immediately below the $\mathrm{P} 2$ region, we have quasiperiodic motion with two limit cycles originated from P2 trajectories. As we penetrate more and more into this black region, we reach a region of chaos. The same happens 


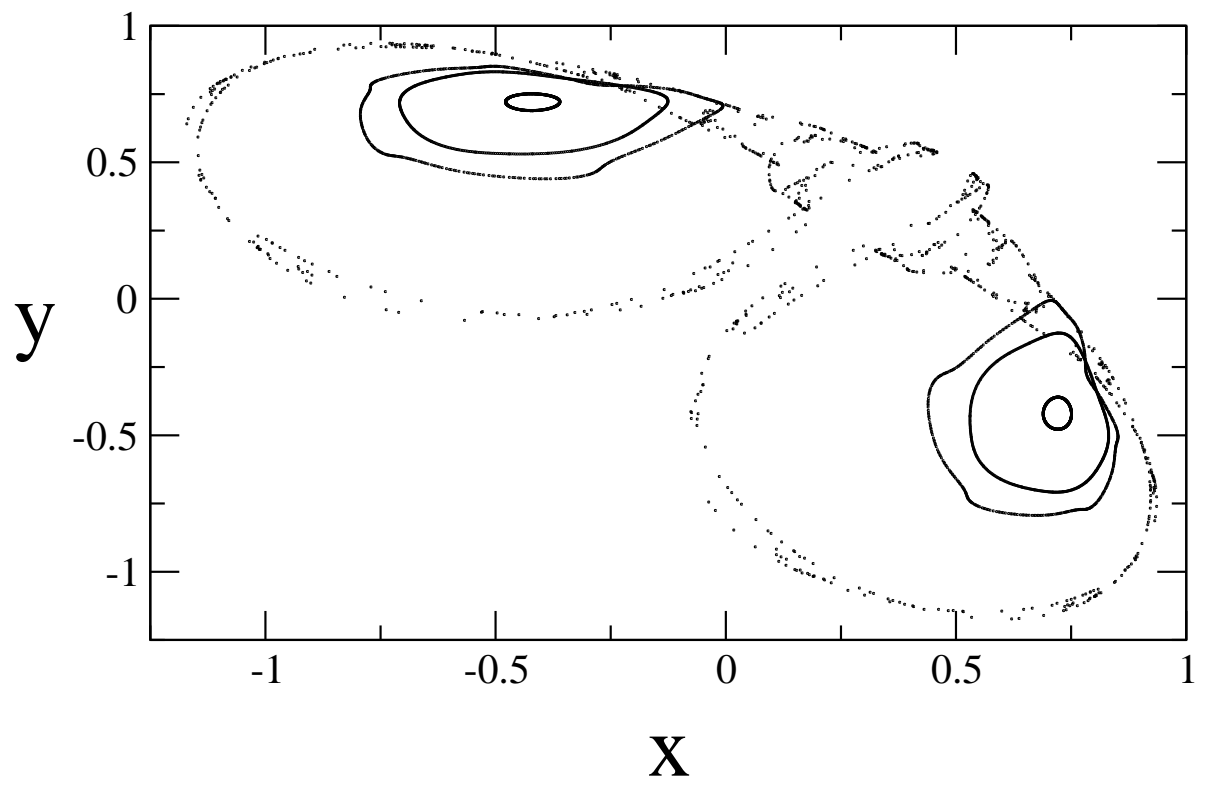

Fig. 3. Quasiperiodicity and chaos seen in the phase-space of two linearly coupled quadratic maps. For $a=0.5$ and $\left(x_{0}, y_{0}\right)=(0.1234,0.005), 1000$ points have been plotted, after a transient of 400 iterations. Shown are three quasiperiodic solutions, for $b=-0.35,-0.38,-0.40$. Here, smaller $b$ corresponds to larger radius. A frequency-locked solution with period- 6 appears at $b=-0.41$ (not shown in the figure). The complicated dotted structure is a chaotic orbit for $b=-0.46$.

for P4 oscillations, the difference in this latter case being that the region of quasiperiodic motions has four limit cycles originated from P4 orbits.

Figure 3 shows the birth and evolution of the Naimark-Sacker bifurcation in phase space when we walk along the vertical line $a=0.5$ in parameter space. Here we plotted the amplitudes of the maps for four representatives values of the parameter $b$. For $b$ close to the Naimark-Sacker line the motion covers a closed curve and is quasiperiodic. For smaller values of $b$, a quasiperiodic transition to chaos takes place. Periodic windows, not visible in Fig. 3, appear in this route to chaos via quasiperiodicity.

We demonstrated that two linearly coupled quadratic maps can show quasiperiodic motion arising from a Naimark-Sacker bifurcation of a P2 orbit. This was shown analytically and corroborated through a numerical simulation, as may be seen from the similarity between Figs. 1 and 2. The first figure was obtained analytically while the second numerically. The precise analytical determination of the Naimark-Sacker road to stable quasiperiodic motions opens the possibility of several numerical experiments, in particular, to applications connected with synchronization in networks of maps $[13,14,15]$. Another inter- 
esting application is to investigate how a mismatch in the parameters would affect the bifurcation structure. These applications will be reported elsewhere.

\section{References}

[1] S. Strogatz, Sync: The Emerging Science of Spontaneous Order, (Hyperion, New York, 2003).

[2] S. Boccaletti, J. Kurths, G. Osipov, D.L. Valladares and C.S. Zhou, Phys. Rep. 366 (2002) 1.

[3] A. Pikovsky, M. Rosenblum, and J. Kurths, Synchronization - A Universal Concept in Nonlinear Sciences (Cambridge University Press, Cambridge, 2001).

[4] Y.L. Maistrenko, V.L. Maistrenko, A. Popovich, and E. Mosekilde, Phys. Rev. E 57 (1998) 2713.

[5] R. V. Buskirk and C. Jeffries, Phys.Rev. A 31 (1985) 3332.

[6] K. Wiesenfeld and P. Hadley, Phys. Rev. Lett. 62 (1989) 1335.

[7] I. S. Aranson, A. V. Gaponov-Grekhov and M. I. Rabinovich, Physica D 33 (1988) 1.

[8] I. Waller and R. Kapral, Phys. Rev. A 30 (1984) 2047.

[9] J. Frøyland, Physica D 8 (1983) 423.

[10] T. Hogg and B. A. Huberman, Phys. Rev. A 29 (1984) 275.

[11] K. E. Kürten and G. Nicolis, Physica A 245 (1997) 446.

[12] H. Kook, F. H. Ling and G. Schmidt, Phys. Rev. A 43 (1991) 2700.

[13] P.G. Lind, S. Titz, T. Kuhlbrodt, J. Corte-Real, J. Kurths, J.A.C. Gallas and U. Feudel, Coupled bistable maps: a tool to study convection parameterization in ocean models, Int. J. of Bif. and Chaos 14 (2004) 999-1015.

[14] P.G. Lind, J. Corte-Real and J.A.C. Gallas, Phys.Rev. E 66 (2002) 016219.

[15] P.G. Lind, J. Corte-Real and J.A.C. Gallas, Physica D 168 (2002) 93.

[16] J. Guckenheimer and P. Holmes, Nonlinear Oscillations, Dynamical Systems, and Bifurcations of Vector Fields (Springer-Verlag, New York, 1983).

[17] S. Wiggins, Introduction to Applied Nonlinear Dynamical systems and Chaos (Springer-Verlag, New York, 1990).

[18] J. A. C. Gallas, Phys. Rev. Lett. 70 (1993) 2714. 